\title{
Jahrringanalytische Untersuchungen in immissionsgefährdeten Waldschadengebieten des Walliser Rhonetales
}

\section{Problemstellung und Ziele}

Der Streit um die Fluorimmissionen im Walliser Rhonetal brachte den stark geschädigten Föhrenwäldern zwischen Martigny und Brig große Publizität. In den Medien wurde oft die Meinung geäußert, daß das auf einer Fläche von rund $130 \mathrm{~km}^{2}$ auftretende Waldsterben allein durch die fluorhaltigen Abgase der Aluminiumfabriken und chemischen Produktionsanlagen verursacht werde. Diese Behauptung kann aus heutiger Sicht nur teilweise bestätigt werden, denn die Ursachen der Waldschäden sind äußerst komplex und auch heute nicht endgültig geklärt. Nebst den nachgewiesenermaßen schädigenden Fluorverunreinigungen können im Wallis verschiedene andere Schadstoffe zu smogähnlichen Verhältnissen führen, welche die Vegetation nachhaltig beeinflussen. Die Wirkungen sämtlicher Luftverunreinigungen werden zusätzlich durch natürliche Streßfaktoren verstärkt. Namentlich die außerordentliche Trockenheit des Walliser Klimas, die starken Talwinde und die flachgründigen Böden an den Talflanken können die Vitalität der Bäume reduzieren und zur Schadenhäufung beitragen. Die Situation wird durch Inversionswetterlagen verschärft, die den Frischluftaustausch reduzieren und zu hohen Schadstoffkonzentrationen in der Grundschicht führen. Die Föhrenwälder wurden zudem an vielen Stellen seit über 20 Jahren nicht mehr genutzt. Überalterung und zum Teil fehlende forstliche Pflege können zur Schadenhäufung beitragen.

Bezüglich der Fluoride traten auf den 16. Oktober 1978 neue kantonale Emissionsvorschriften in Kraft. Im Hinblick auf diese strengen Verordnungen wurden in allen Aluminiumhütten des Walliser Rhonetals moderne Filteranlagen installiert, so daß der Fluoraussto $ß$ heute unter dem Grenzwert von 1,5 kg Fluor pro Tonne Aluminium liegt. Vor dem Einbau der Filteranlagen lag die Fluorbelastung in gewissen Talabschnitten weit über den tolerierbaren Grenzen. Messungen, die von der Eidgenössischen Anstalt für das forstliche Versuchswesen (EAFV) in den Jahren 1977 und 1978 durchgeführt wurden, zeigten, daß die Region Chippis damals sehr stark belastet war, während das Gebiet von Martigny in den späten siebziger Jahren sehr geringe Werte aufwies. Hingegen war die Belastung durch die Schadstoffe $\mathrm{SO}_{2}, \mathrm{HCl}, \mathrm{NO}_{\mathrm{x}}$ und andere, möglicherweise nicht erfaßte Komponenten zwischen

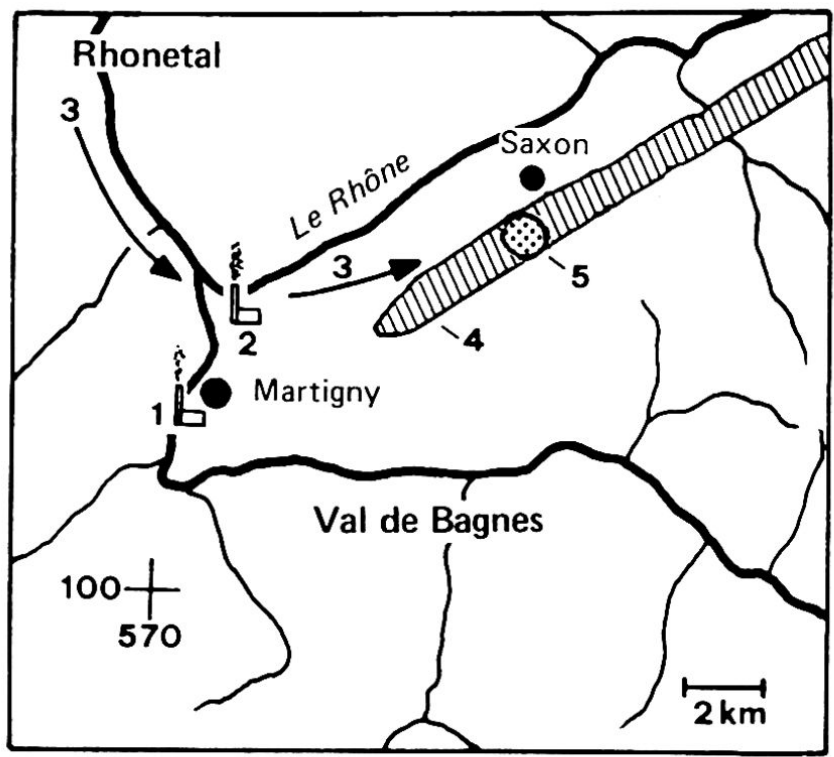

Abb. 1 Karte des Untersuchungsgebietes.

(1) Aluminiumproduktion von 1907-1956

(2) Aluminiumproduktion seit 1938 (Produktionsunterbruch 1940-1945)

(3) Hauptwindweg bei W- und NW-Windlage

(4) Gebiete mit starken Waldschäden

(5) Jahrringanalytisch untersuchte Waldbestände

Martigny und dem Genfersee höher als im Gebiet von Chippis. Auf Grund der Immissionssituation der späten siebziger Jahre ist der Fluoreinfluß im Untersuchungsgebiet von Martigny sehr umstritten, während die Verhältnisse in den oberen Talabschnitten klarer sind. Ältere Gutachten und wissenschaftliche Arbeiten zeigen jedoch deutlich, daß die landwirtschaftlichen Kulturen in der Gegend von Martigny in früheren Jahren unter beträchtlichen Fluorimmissionen gelitten haben. Ob dies für die Waldpartien ebenfalls zutrifft, muß retrospektiv geprüft werden. Die Jahrringanalyse erweist sich dabei als geeignete Methode, um die Bestandesgeschichte der geschädig-

Felix Kienast, dipl. Geogr., Eidgenössische Anstalt für das forstliche Versuchswesen, $\mathrm{CH}-8903$ Birmensdorf $\mathrm{ZH}$ 
ten Wälder zu erforschen. Mit Hilfe der Jahrringe können Ereignisse nachgewiesen werden, die Jahre oder gar Jahrzehnte zurückliegen. Die Hauptziele der vorliegenden Arbeit bestanden deshalb darin, im Jahrringbild den Zeitpunkt einer allfälligen Schädigung festzustellen und die schadenbedingten $\mathrm{Zu}$ wachsverluste abzuschätzen.

\section{Die untersuchten Waldbestände}

Die jahrringanalytisch untersuchten Waldbestände liegen oberhalb Saxon auf zirka 800 bis 900 Meter über Meer (siehe dazu Abbildung 1). Der Hang ist allgemein gegen Nordwest exponiert und weist eine durchschnittliche Neigung von zirka 50 Prozent auf. Die absterbenden Bestände liegen hauptsächlich am Prallhang des häufig talaufwärts wehenden Windes, in dessen Einzugsgebiet der Fluoremittent seit dem Jahre 1938 steht (Produktionsunterbruch 1940-1945). Die Standorteigenschaften der sechs untersuchten Waldbestände wurden durch pflanzensoziologische und bodenkundliche Aufnahmen erfaßt. Die Föhrenwälder können danach zum größten Teil den von ELLENBERG und KLÖTZLI (1972) beschriebenen Waldgesellschaften des Geißklee-Föhrenwaldes und des Schneeheide-Föhrenwaldes zugeordnet werden. Eine Ausnahme bildet ein in der Schattenlage einer ausgeprägten Hangrinne gelegener, ungeschädigter Waldbestand (Nr.4, Abbildung 5). Er steht dem Zwergbuchs-Fichtenwald nahe.

Das Probenmaterial setzte sich aus 391 Bohrkernen und 37 Stamm- und Astscheiben zusammen. Die noch stehenden Bäume wurden auf Brusthöhe mit $\mathrm{Zu}-$ wachsbohrern angebohrt, während von den toten, liegenden Bäumen Stammscheiben entnommen wurden.

\section{Untersuchungsmethoden}

\subsection{Okulare Datierung mit Hilfe von Weiserjahren}

Mit der nachstehend beschriebenen, einfachen Datierungsmethode wurden zirka 75 Prozent des Probenmaterials zweier Waldbestände rationell und zielgerichtet verarbeitet. In der Abbildung 2 ist das Vorgehen graphisch dargestellt. Die Methode stützt sich auf die ökophysiologische Tatsache, daß an Trockenstandorten des Wallis der radiale Zuwachs, beziehungsweise die kambiale Aktivität der Bäume hauptsächlich durch die Sommerniederschläge gesteuert wird (sCHWEINGRUBER et al. 1979). In Trockenjahren werden nur ganz schmale Jahrringe und schwache Spätholzzonen gebildet. Solche Jahrringe werden Weiserjahre genannt. Im Walliser Trockengebiet treten sie in den letzten hundert Jahren in genügend dichter Zahl auf, so daß die Bohrproben ohne Jahrringbreitenmessung datiert werden konnten. Anschließend wurden aus dem Jahrringbild drei Ereignisse zeitlich festgelegt; der Beginn des kambialen Wachstums, eine allfällige Hemmung und das Ende der kambialen Aktivität. Die Daten wurden nachher statistisch ausgewertet, um allfällige Schadeneinflüsse zeitlich zu erfassen.

\subsection{Radiodensitometrische Jahrringanalyse}

Rund ein Viertel des Probenmaterials wurde radiodensitometrisch untersucht. Das Verfahren, das von LENZ et al. (1976) beschrieben wurde, liefert für jede Jahrringsequenz ein kontinuierliches Holzdichteprofil, wie es in der Abbildung 3 dargestellt ist. Aus der Dichtekurve werden pro Jahrring fünf Parameter digital erfaßt und als Zeitreihe aufgezeichnet. Die Jahrringsequenzen werden anschließend mit Hilfe verschiedener statistischer Tests und bereits bestehender Chronologien synchronisiert.

unterdrückte Jugendphase

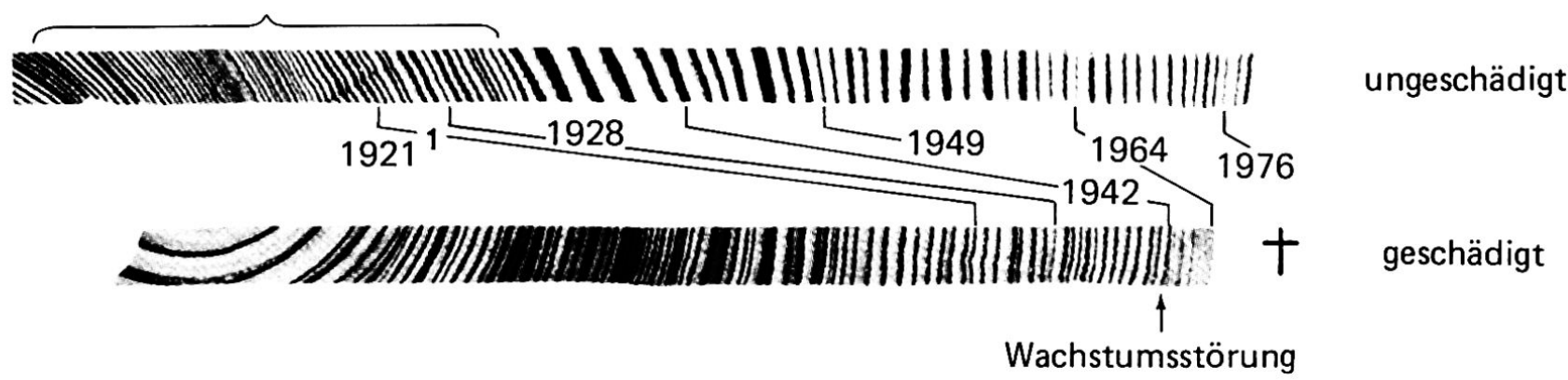

Abb. 2 Okulare Datierung mit Hilfe von Weiserjahren am Beispiel zweier radiographierter Bohrkerne von Waldföhren (Pinus silvestris L.) aus dem Untersuchungsgebiet bei Saxon. (1) = ohne Binokular nicht erkennbare Weiserjahre. 


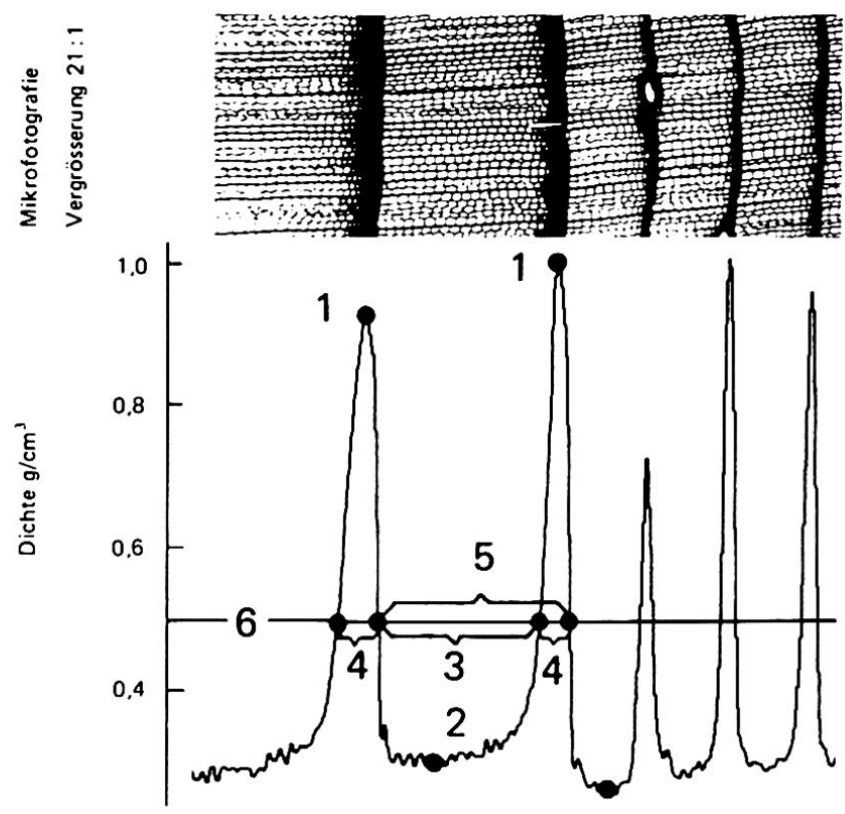

Abb. 3 Mikrophotographie und zugehöriges Dichtediagramm einer Jahrringabfolge aus Lärchenholz (Larix decidua Mill.).

(1) Maximale Spätholzdichte

(2) Minimale Frühholzdichte

(3) Frühholzbreite

(4) Spätholzbreite

(5) Jahrringbreite

(6) Schwellenwert Frühholzbreite/Spätholzbreite

(nach SCHWEINGRUBER et al. 1979)

\section{Eliminierung des Alterstrends}

Jeder Baum unterliegt während seines Wachstums dem Prozeß der Alterung. Nach einer anfänglichen Jugendphase, in der das Wachstum einen Höhepunkt erreicht, sinkt die Wuchsleistung mit zunehmendem Alter ab. Der Alterstrend zeigt sich im Jahrringbild am deutlichsten in einer Abnahme der Jahrringbreite mit zunehmendem Alter. Dieser störende Einfluß mußte bei den vorliegenden Jahrringsequenzen eliminiert werden, damit der altersbedingte Zuwachsrückgang nicht als anthropogener Schaden interpretiert wird. An der EAFV stehen Computerprogramme zur Verfügung, die es erlauben, den Alterstrend mit verschiedenen mathematischen Zuwachsfunktionen auszugleichen und dem individuellen Wachstum eines Baumes anzupassen. Bei den vorliegenden Jahrringkurven wurde die Zuwachsfunktion während einer möglichst langen, immissionsfreien Periode geeicht und anschließend in den beeinflußten Zeitabschnitt extrapoliert. Die immissionsfreie Periode wurde bis und mit dem Jahre 1940 festgelegt, obwohl bereits in den späten dreißiger Jahren Fluoreinflüsse vorhanden waren. Während der Extrapolationsphase wird dadurch ein möglicher Schadeneinfluß höchstens zu gering abgeschätzt, da noch mögliche Schadenjahre in die Alterstrendberechnung eingehen. Würde die Eichperiode stärker verkürzt, ergäbe sich eine sehr lange Extrapolationsperiode, was zu Extrapolationsfehlern führen könnte. Mit dieser Kompromißlösung erstreckte sich die Extrapolationsperiode über knapp 40 Jahre, was den von pollansChüTz (1971) und NEUMANN Und SCHIELER (1981) angegebenen Zeitraum überschreitet. Um Fehlextrapolationen zu vermeiden, wurden die Ausgleichsfunktionen sämtlicher Einzelkurven und Jahrringparameter auf einem Bildschirm visualisiert und Fehlextrapolationen ausgeschieden.

\section{Ermittlung der Beziehungen zwischen der Witterung und dem Jahrringwachstum}

Um einen anthropogen bedingten Zuwachsrückgang von klimatischen Einwirkungen $\mathrm{zu}$ unterscheiden, wurden die Korrelationen zwischen den Witterungsdaten und dem Jahrringzuwachs während der immissionsfreien Periode ermittelt. Anschließend erfolgte die Schätzung der Jahrringparameter auf Grund der Klimadaten während der ganzen Periode, die mit klimatischen Messungen dokumentiert ist. Die Abweichungen zwischen den geschätzten und den gemessenen Jahrringkurven sollten sich bei gesunden Bäumen im Mittel aufheben. Systematische Abweichungen zwischen den beiden Kurven müssen auf nichtklimatische Fremdeinflüsse zurückzuführen sein. Als Witterungsdaten dienten die Monatsmittel der Temperatur und des Niederschlags der meteorologischen Stationen Sion und Grand St. Bernhard. Die angewendete Regressionsanalyse wurde von KIENAST (1982) beschrieben und mit den Resultaten der multiplen Regressionsanalyse nach FRITTS (1976) verglichen. Die beiden Methoden ergaben sehr ähnliche Resultate.

\section{Ergebnisse}

\subsection{Okulare Datierung mit Hilfe von Weiserjahren}

Mit Hilfe der einfachen, zeitsparenden Methode wurde eine hohe Datierungsquote erreicht. Es gelang, 86 Prozent der Bohrkerne und 75 Prozent der Stammund Astscheiben zweier Waldbestände zeitlich einzuordnen. Auf Grund der statistischen Auswertung der drei erfaßten Ereignisse Beginn, Hemmung und Ende der kambialen Aktivität steht fest, $\mathrm{da} ß$ sich die Wachstumsstörungen in den vierziger Jahren dieses Jahrhunderts markant häufen (siehe dazu Abbildung 4). Dieses Ergebnis ist umso erstaunlicher, als das Baumalter der untersuchten Individuen sehr variabel ist. Die Hauptschadenjahre 1942, 1944 und 1947 treten sowohl bei ausschließlich jungen wie bei sehr alten, bereits abgestorbenen und vermoderten Bäumen als Hauptschadenjahre auf. Das Ende der kambialen Aktivität häuft sich in den frühen siebziger Jahren und fällt meistens mit Trockenperioden zusammen. Der Höhepunkt der Absterbeperiode scheint aber noch nicht überschritten zu sein. 


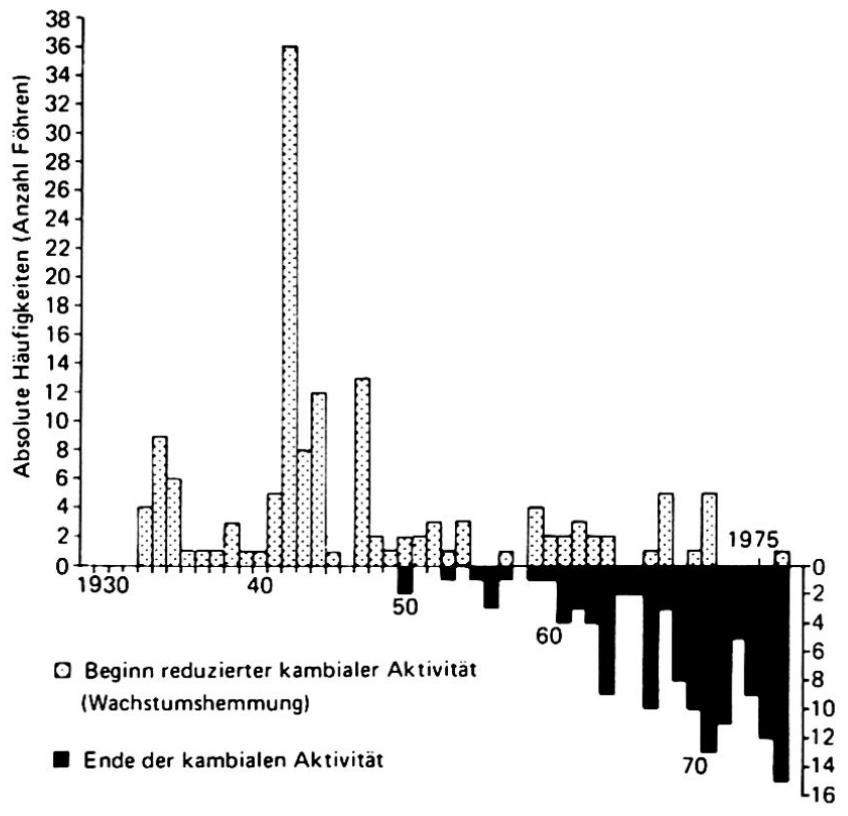

Abb. 4 Häufigkeitsverteilung von zwei datierten Ereignissen; Wachstumshemmung und Ende der kambialen Aktivität. Für jedes Kalenderjahr ist die Anzahl der auf dieses Jahr datierten Probebäume aufgetragen|(nach KIENAST et al. 1981).

\subsection{Radiodensitometrische Jahrringanalysen}

Die Resultate der okularen Datierung wurden durch die radiodensitometrischen Untersuchungen weitgehend bestätigt. In der Abbildung 5 sind die indexierten (alterstrendfreien) Mittelkurven der maximalen Spätholzdichte für verschiedene Waldbestände und Schadstufen dargestellt. Der Wachstumsrückgang ist bei den geschädigten Beständen klar ersichtlich. Bei den mittel geschädigten Bäumen ist der Beginn reduzierter kambialer Aktivität um 1960, bei den stark geschädigten Waldbeständen bereits in den vierziger Jahren anzusetzen. Alle geschädigten Waldbestände, die untersucht wurden, verhalten sich hinsichtlich zeitlichen Auftretens der Wachstumshemmung gleich. Die Jahrringbreite, die nicht dargestellt wurde, ergab die gleichen Resultate.

Im Jahrringbild zeigt sich der Einflu $\beta$ des Standorts sehr stark. Nur noch die extremsten Trockenjahre des Jahrhunderts wie 1921 und 1976 können in allen Waldbeständen als Weiserjahre lokalisiert werden. Auf Grund der multiplen Regressionsanalyse nach FRITTS (1976) führen in den Trockenstandorten hohe Frühsommerniederschläge kombiniert mit tiefen Spätsommertemperaturen zu einer hohen Assimilationsleistung. In den Probeflächen mit feuchten Wachstumsbedingungen erklären Niederschlag und Temperatur den Jahrringzuwachs schlecht. Möglicherweise würde man mit der potentiellen oder aktuellen Evapotranspiration bessere Resultate erreichen.

Um den anthropogen bedingten Schaden abzuschätzen, wurden sowohl den Einzel- wie den Mittelkurven die fiktiven Jahrringkurven überlagert, die mit Hilfe der Klimadaten errechnet wurden. In der Abbildung 6 sind zwei Beispiele für die Einzelkurven der maximalen Dichte dargestellt. Mit Hilfe des t-Tests wurde bei sämtlichen Einzelbäumen geprüft, ob die berechneten Werte signifikant von den gemessenen Werten abweichen. Durchschnittlich ist der Schaden beim Parameter Spätholzbreite am deutlichsten feststellbar, gefolgt von der Jahrringbreite, der Frühholzbreite und der maximalen Spätholzdichte. Letztere reagiert offenbar träge auf nicht klimatische Einflüsse. Die Zuwachsverluste der geschädigten Bestände wurden auf die Extrapolationsperiode von 1941 bis 1979 bezogen. Gegenüber der berechneten, fiktiven Wachstumsleistung verzeichnet man in dieser Periode rund 20 bis 30 Prozent weniger Radialzuwachs und 30 bis 40 Prozent weniger Spätholzbreitenzuwachs. Gesunde Bäume sind dabei miteingeschlossen und zuwachsvermindernde Einflüsse wie Trockenheit und Alterung bereits berücksichtigt.

\section{Interpretation der Ergebnisse}

Die Analyse der Jahrringkurven zeigte, daß ein Schadeneinfluß vorliegt, der nicht primär auf die natürlichen, wachstumsvermindernden Faktoren wie Trockenheit und Alterung zurückzuführen ist. Die geschädigten Bäume erreichen während der immissionsgefährdeten Periode in praktisch keinem Jahr den klimatisch und altersmäßig möglichen Zuwachs, wie er noch anfangs des 20. Jahrhunderts festzustellen war. Zusätzliche Streßfaktoren müssen das Wachstum eingeschränkt haben. Ob die Waldbestände durch die Fluorimmissionen der Aluminiumfabrik Martigny geschwächt wurden, kann nicht kausal nachgewiesen werden. Die folgenden Interpretationen sind deshalb als Indizien zu werten. Sie zeigen aber deutlich, daß der Fluoreinfluß eine stichhaltige Erklärung der Ergebnisse ist. Vor 1938 lag die Aluminiumfabrik Martigny abseits des Haupttales am Eingang des Val de Bagnes. Im Jahre 1938 wurde die Produktion in die neue Anlage im Hauptwindbereich des Rhonetals verlegt, wo der Betrieb von 1940 bis 1945 eingestellt blieb. Die Verlegung mußte die Immissionssituation tiefgreifend verändert haben, denn in der darauffolgenden Trockenperiode (1942 bis 1944) stellt man anhand der Jahrringkurven erste Wachstumshemmungen fest. Die Fluorverbindungen wurden damals ungefiltert an die Umgebung abgegeben. Deshalb genügte bereits die kurze Immissionsperiode der späten dreißiger Jahre, um die Bäume derart zu schwächen, daß sie den natürlichen Streßfaktoren nicht mehr gewachsen waren. Nach der erneuten Produktionsaufnahme im Jahre 1945 folgten im Trockenjahr 1947 weitere Schädigungen. Viele geschädigte Bäume wiesen bis in die siebziger Jahre eine 


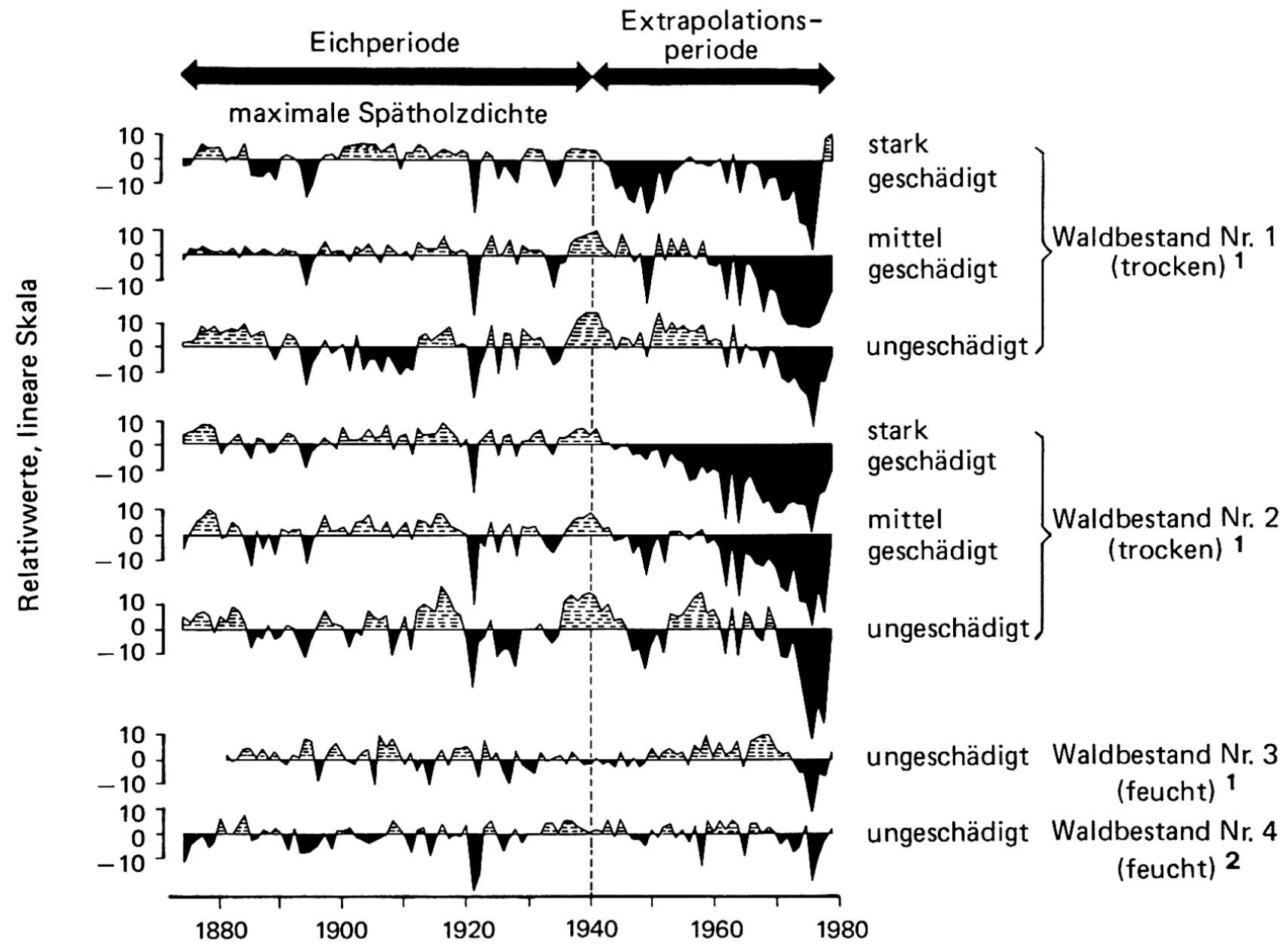

Abb. 5 Alterstrendfreie Mittelkurven der maximalen Spätholzdichte verschiedener Waldbestände des Untersuchungsgebietes. Die Bäume wurden auf Grund terrestrischer Vitalitätsansprachen in verschiedene Schadgruppen eingeteilt. Holzarten: (1) Waldföhre (Pinus silvestris L.), (2) Fichte (Picea abies Karst.).

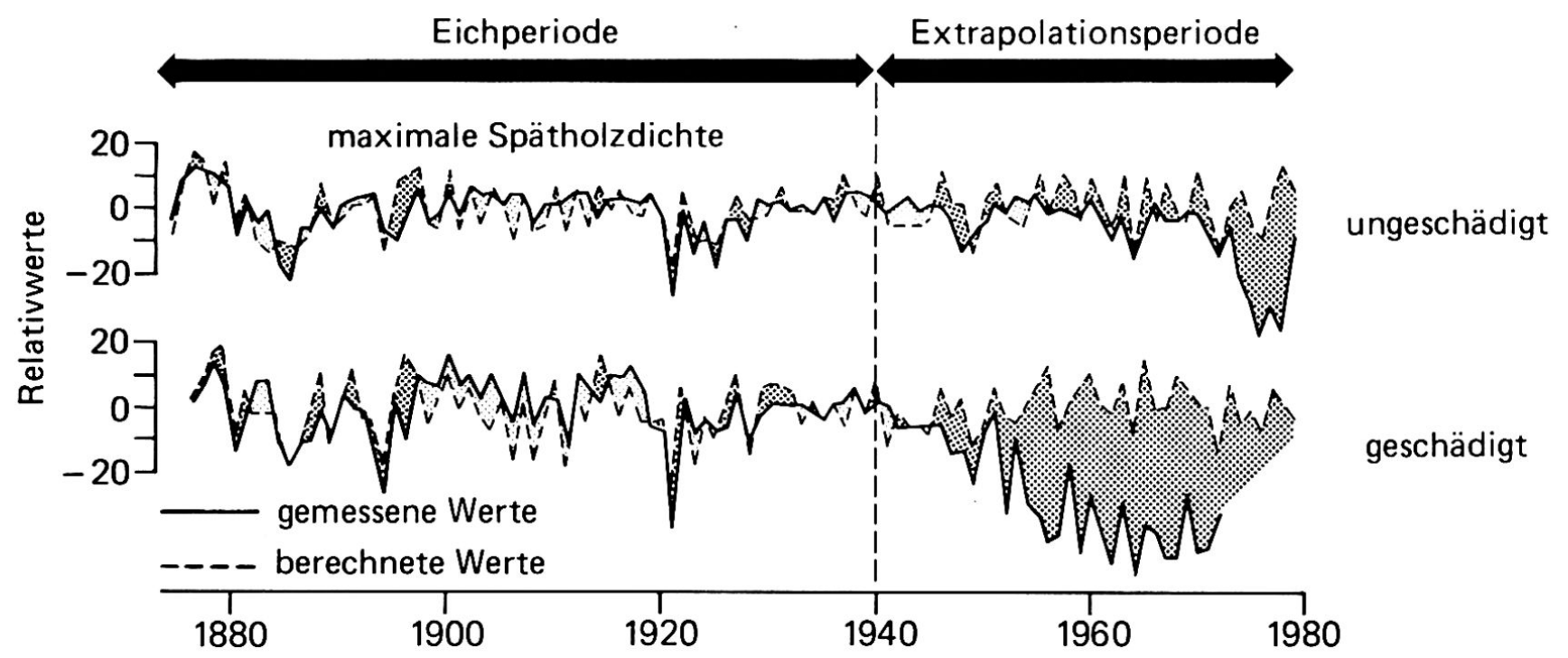

Abb. 6 Abschätzung des Schadens am Beispiel der indexierten Einzelkurven der maximalen Spätholzdichte zweier Waldföhren (Pinus silvestris L.) aus dem Untersuchungsgebiet bei Saxon. Die fiktiven Kurven, welche die gemessenen Werte überlagern, wurden mit Hilfe von Klimadaten berechnet. 
immer schwächere Wachstumsleistung auf und starben schließlich endgültig ab. Die künftige Bestandesgeschichte der schwach geschädigten Wälder und der Neuaufforstungen wird zeigen, ob die heute nachweisbar zurückgegangene Fluorbelastung zu einer gesundheitlichen Erholung der Walliser Waldbestände führt.

\section{Summary}

The pine forests of the Rhone Valley are severely damaged. Possible causative agents are fluoride and other harmful gases such as $\mathrm{SO}_{2}, \mathrm{HCl}$ and $\mathrm{NO}_{\mathrm{x}}$ in the emissions of nearby aluminium smelters and chemical plants. Drought and aging may also have contributed to the damage. Core samples and trunk discs from damaged and undamaged stands near Martigny were investigated using annual ring analysis. After visual dating of the samples by means of "pointer» rings formed during dry years, growth disturbances were pinpointed. Radiodensitometrical measurements followed, and age trends were eliminated using mathematical growth functions. A model of the relationship between annual ring, temperature and precipitation was constructed for the emission-free period 1874-1940 and extrapolated to give the values expected for the last hundred years if growth were influenced by climatic factors only. Annual increments for the period 1941-1979 were $20-30 \%$ below the expected value, regardless of tree age, indicating a harmful factor not primarily related to drought or aging. Growth disturbances occurred most commonly during the droughts of the forties and may be regarded as resulting from the emissions which began in 1938 when a new aluminium smelter was built in the main valley: it stood in the mainstream of the prevailing winds and was without filters until 1965. The harmful emissions of the late thirties may have damaged the trees to such an extent that they could no longer withstand natural stresses and finally died $30-40$ years later. Although the fluoride emissions are not causally related to the forest damage, they provide a valid explanation of the results.

\section{Verdankung}

Der vorliegende Beitrag geht aus einer Diplomarbeit hervor, die am Geographischen Institut der Universität Zürich unter der Leitung von Herrn Prof. Dr. G. Furrer und Herrn PD Dr. F.H.Schweingruber ausgeführt wurde. Weiter haben zum Gelingen beigetragen: Frau M.J.Sieber (Übersetzung der Zusammenfassung ins Englische) sowie die Herren Dr. H. Flühler, Dr. O. U. Bräker und E. Schär. Ihnen allen sei hiermit herzlich gedankt.

\section{Literatur}

ECKSTEIN, D., BREYNE, A., ANIOL, R.W., LIESE, W. (1981): Dendroklimatologische Untersuchungen zur Entwicklung von Straßenbäumen. In: Forstw. Cbl., Nr. 100/6, 381-396.

ELLENBERG, H., KLÖTZLI, F. (1972): Waldgesellschaften und Waldstandorte der Schweiz. In: Mitt. Eidg. Anst. forstl. Versuchswes., Nr. 48/4, 587-930.

FRITTS, H. C. (1976): Tree rings and climate, Academic Press, London, New York, San Fancisco.

KIENAST, F., FLÜHLER, H., SCHWEINGRUBER, F.H. (1981): Jahrringanalysen an Föhren (Pinus silvestris L.) aus immissionsgefährdeten Waldbeständen des Mittelwallis (Saxon, Schweiz). In: Mitt. Eidg. Anst. forstl. Versuchswes., Nr. 57/4, 415-432.

KIENAST, F. (1982): Jahrringanalysen in immissionsgefährdeten Waldschadengebieten des Mittelwallis (Saxon). Unpubl. Diplomarbeit Geogr. Inst. Univ. Zürich.

LENZ, O., SCHÄR, E., SCHWEINGRUBER, F.H. (1976): Methodische Probleme bei der radiographisch-densitometrischen Bestimmung der Dichte und der Jahrringbreiten von Holz. In: Holzforschung Nr. 30, 114-123.

NEUMANN, M., SCHIELER, K. (1981): Vergleich spezieller Methoden zuwachskundlicher Schadensabschätzung. In: Mitt. d. forstl. Bundes-Versuchsanst. Wien, Nr. 139, 49-65.

POLLANSCHÜTZ, J. (1971): Die ertragskundlichen Meßmethoden zur Erkennung und Beurteilung von forstlichen Rauchschäden. In: Mitt. d. forstl. Bundes-Versuchsanst. Wien, Nr.92, 153-206.

SCHWEINGRUBER, F.H., BRÄKER, O.U., SCHÄR, E. (1979):

Dendroclimatic studies on conifers from central Europe and Great Britain. In: Boreas Nr. 8, 427-452. 